\title{
ANÁLISIS DEL RIESGO EN UN MODELO MULTINIVEL DE SUMINISTRO
}

\author{
Jesús Escalante, Ileana Camila Monsreal Barrera, \\ Alan García LiRA, Jorge SANTOS Flores \\ Universidad Politécnica de Cataluña \\ JOSEP CASANOVAS-GARCIA* \\ Universidad Politécnica de Catalunya-Barcelona Tech
}

Recibido: 29 de marzo del 2019 / Aprobado: 21 de julio del 2019

doi: 10.26439/ing.ind2019.n037.4541

RESUMEN: La investigación propone las pruebas de estrés para evaluar un conjunto de escenarios hipotéticos de crisis vinculados a periodos con mayor volatilidad, dada una muestra de transacciones comerciales entre tres entidades de un modelo multinivel de suministro. La solución identifica los niveles de impacto en términos de costo para un conjunto de escenarios hipotéticos asociados a parámetros tales como niveles de confiabilidad y volatilidad en un horizonte temporal de riesgo.

Palabras clave: riesgo / prueba de estrés / modelo multinivel / simulación de Monte Carlo

\section{RISK ANALYSIS IN A MULTILEVEL SUPPLY MODEL}

ABSTRACT: This research proposes stress tests to evaluate a set of hypothetical crisis scenarios linked with periods of greater volatility in a sample of commercial transactions among three companies from a multilevel supply model. The solution identifies cost-related levels of impact for a set of hypothetical scenarios associated with parameters such as levels of reliability and volatility in a risky time horizon.

Keywords: risk / stress test / multilevel model / Monte Carlo simulation

\footnotetext{
*jesus.escalante@correo.uady.mx, ileana.monsreal@correo.uady.mx, glira@correo.uady.mx, sflores@correo.uady.mx, josepk@fib.upc.edu
} 


\section{INTRODUCCIÓN}

\subsection{Contexto}

Yucatán es una de las diez regiones económicas de México con mayor crecimiento en los últimos años de acuerdo a las cifras del Organización para la Cooperación y el Desarrollo Económico (OCDE), clasificada con las condiciones favorables para continuar su crecimiento (OCDE, 2008).

Por otra parte, el Instituto Nacional de Estadística y Geografía de México (INEGI, 2009) señala que en este estado las pymes representan el $99,6 \%$.

Mérida, la capital del estado, concentra la mayor parte de la actividad industrial y comercial, con una cifra del $42 \%$ de la población; la tasa de ocupación es del $60 \%$, con producción de más de dos tercios del producto bruto regional (INEGI, 2014).

La ciudad de Mérida es favorable dada su relación con el resto de la península de Yucatán y con Centroamérica. De acuerdo a las características de ubicación geográfica, infraestructura física y tecnológica, es considerada como una de las regiones con mayor potencial de desarrollo para las actividades de logística, distribución y fabricación de componentes para Centroamérica, Sur de México, el Caribe y los Estados Unidos.

No obstante, los informes de la OCDE refieren que Mérida y Yucatán muestran grandes rezagos que, aunados a bajos niveles de capital humano, falta de innovación y redes de suministro insuficientes y poco competitivas la hacen vulnerable. Estudios del Banco Interamericano de Desarrollo (González et al., 2014) refieren dos indicadores importantes: el Índice de Exposición de Desastres (IED) para medir la vulnerabilidad, así como el índice de Exposición a Eventos Climáticos Extremos (ECE).

Estimaciones del IED indican la posibilidad de un escenario inesperado para las regiones que habían sido consideradas resilientes y que se encuentran cada vez más expuestas. El $45 \%$ de los desastres ocurrieron en Centroamérica y países más poblados, como Colombia y México, son los que tienen más alta participación. De acuerdo con información de la Agenda de la Organización de las Naciones Unidas, las inundaciones y deslizamientos de tierra han sido los sucesos predominantes (González et al., 2014).

\subsection{Enfoque metodológico}

Entre los métodos alternos para el análisis de escenarios están las pruebas de estrés. Este enfoque supone que deberá valorarse aquella entidad que podría sufrir pérdidas importantes asociadas a riesgos excepcionales. La investigación consideró como objetivo valorar un conjunto de escenarios hipotéticos de crisis asociados a los efectos disruptivos vinculados a periodos con mayor volatilidad para una muestra de transacciones comerciales entre tres entidades configuradas como modelo multinivel de suministro. 
Los escenarios corresponden a cuatro estadios entre las transacciones comerciales, siendo el estatus normal (N) entregas a tiempo (T), anticipadas (A) y con demora (D). La motivación ha sido estimar la pérdida potencial que podría sufrir el sistema considerando las condiciones referidas. En los últimos años el tema ha tenido un especial interés, por ejemplo en la evaluación del riesgo en cadenas de suministro (Heckmann, Comes y Nickel, 2014) o para tener una idea de los efectos de escenarios adversos sobre una determinada cartera de inversión. Otro enfoque es el análisis del VaR histórico en el que, a partir del marco temporal histórico elegido, es posible estimar la distribución de probabilidad empírica de los cambios en las variables del mercado. Generalmente los cambios de cada día son igualmente ponderados y la elección de la ponderación se define arbitrariamente (Meucci, 2010).

El concepto de Value at Risk (VaR) (Jorion, 2010; Olson y Wu, 2017) tiene la finalidad de cuantificar, con un nivel de incertidumbre, la pérdida en un horizonte de planeación. Este se define como un valor límite tal que la probabilidad de que una pérdida a precios de mercado en la cartera sobre el horizonte temporal dado exceda ese valor (asumiendo mercados normales y que no se produce negociación en la cartera); para más detalles sobre este modelo véase Boucher, Danielsson, Kouontchou y Maillet (2014). Para entornos industriales, estimar el VaR con un nivel de significancia del $5 \%$ implicaría que, una vez al mes, el retorno de las inversiones (para este caso los impactos de las transacciones comerciales) se vea afectado más de lo que indique el VaR en relación con el retorno esperado.

La investigación propone dos enfoques para evaluar el riesgo (VaR). El primero considera un método alterno para el análisis de escenarios mediante pruebas de estrés. Este método supone que deberá valorarse aquella entidad que podría sufrir pérdidas por riesgos excepcionales. La prueba de estrés debe utilizarse como una técnica complementaria (Shekh y Marsh, 2016; Abdymomunov y Gerlach, 2014) con el fin de garantizar que la entidad sea capaz de cubrir las pérdidas incluso si la organización se enfrentará a eventos de mayor riesgo. El segundo enfoque incorpora los datos históricos como elementos de análisis para simular los tres estados del sistema y valorar su impacto como efecto de las disrupciones utilizando la simulación de Monte Carlo.

\section{DESCRIPCIÓN DEL SISTEMA}

\subsection{Modelo multinivel de suministro}

Se ha considerado un enfoque multinivel para evaluar el comportamiento del sistema, con una aproximación integral a través de toda la red (figura 1), teniendo en cuenta el impacto en los costos asociados a las transacciones comerciales en los tres niveles del sistema. El enfoque ha delimitado la estructura a tres componentes: minoristas (M), centros de distribución (CD) y proveedores (P). 


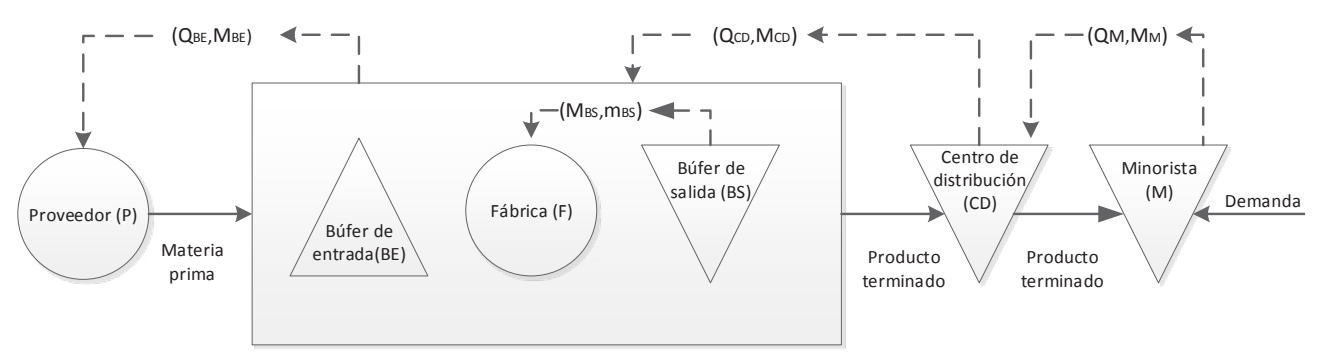

Figura 1. Modelo multinivel

Fuente: Altiok y Melamed, 2007

El minorista se enfrenta a un flujo de demanda por parte de los clientes finales. Para gestionar el inventario utiliza una política de control continuo del stock (Q, M). Ante esta política, la reposición del inventario $Q$ desde el centro de distribución se realiza cuando los niveles de inventario para el minorista sean necesarios (órdenes de inventario disponibles menos pedidos pendientes).

Si el centro de distribución cuenta con suficiente stock, el tiempo de entrega del pedido se compone de los retrasos en el transporte y las inexistencias hacia niveles superiores del centro de distribución. El flujo de órdenes para el centro de distribución proviene del minorista.

Sin embargo, a diferencia del centro de distribución, la demanda insatisfecha quedará pendiente; el centro de distribución repone sus reservas desde el búfer de salida (BS) de la fábrica hacia el nivel superior de la cadena a base de una política de revisión continua del inventario $\left(Q_{C D}, M_{C D}\right)$. La cantidad no satisfecha de los pedidos realizados quedará en estado pendiente.

La política de fabricación se basa en una revisión continua del stock ( $\left.M_{B S}, m_{B S}\right)$. La fábrica produce un producto por unidad de tiempo, después de haber consumido una unidad de materia prima a partir del stock intermedio de entrada. Ante la escasez de materia prima en este búfer de entrada, se detendría la producción.

El búfer de entrada corresponde a los pedidos que son entregados por un proveedor externo con inventarios ilimitados. El plazo de entrega de materia prima se limita a la demora del transporte cuando las órdenes en fábrica estén plenamente satisfechas, siendo una política de control de inventario continua ( $\left.Q_{B E}, M_{B E}\right)$. El sistema está sujeto a los siguientes supuestos:

1. El minorista se enfrenta a la demanda que llega a razón de una unidad por unidad de tiempo, donde todas las demandas insatisfechas se pierden. 
2. En todos los niveles los pedidos se reciben en el orden en que fueron solicitados (sin órdenes adelantadas). Es decir, se solicita un pedido solo cuando el anterior se encuentra en su destino.

3. Cuando se produce el desabasto en el centro de distribución o en la fábrica, la proporción no satisfecha será una orden pendiente (orden atrasada). Una orden satisfecha estará dada cuando el pedido completo esté disponible. En síntesis, no hay envío de órdenes parciales.

\subsection{Configuración del modelo}

Para esta etapa se fraccionó el modelo en pequeños componentes con el fin de verificar que las actividades corresponden a una red típica de suministros en una pequeña o mediana empresa. Se procuró una intensa comunicación con el Centro de Emprendimiento e Innovación Tecnológica (CEINTEC de México) por sus valiosas aportaciones para la configuración del modelo conceptual.

\section{METODOLOGÍA}

Dado que las pruebas de estrés a menudo definen los eventos con una muy baja probabilidad de ocurrencia y, en consecuencia, los resultados se vuelven difíciles de interpretar, se ha propuesto una selección de escenarios que permiten su valoración.

Los escenarios pueden dividirse en dos grupos basados en el tipo de evento. El primero utiliza eventos históricos causales (desastres naturales, eventos terroristas, etcétera) por ejemplo el huracán Katrina (Bueno-Solano y Cedillo-Campos, 2014; Escalante, Casanovas y Monsreal, 2016). El segundo grupo, más ampliamente utilizado en la práctica, utiliza escenarios hipotéticos. Los escenarios se basan en eventos de riesgo que no han sucedido aún, pero con cierta probabilidad de ocurrencia (Flood y Korenko, 2015).

Un escenario típico consiste en la descripción del estado complejo que impondría un evento de riesgo extremo a una entidad entre sus elementos: las probabilidades y frecuencias de ocurrencia, las pérdidas generadas por el evento y las estrategias de mitigación.

A pesar de que el escenario pretende ser realista, no es posible incluir todos los factores y sus características de riesgo. Sin embargo, los gestores de riesgo están tratando de definir los escenarios de modo que correspondan a la realidad tanto como sea posible (Alexander y Sheedy, 2008; Jorion, 2003). En síntesis, se pretende obtener una visión general sobre los eventos de baja frecuencia que puedan tener graves repercusiones entre entidades empresariales; en nuestro caso, el modelo multinivel de suministro. 
Las pérdidas generadas por un conjunto de cuatro escenarios fueron agregadas a las estimaciones de capital basadas en la muestra de datos original, utilizando el método VaR. Los resultados se analizan a continuación.

\subsection{Estructura de los datos}

La estructura de costos se compone de una muestra total de 4500 observaciones y costos globales por día, dado un horizonte mensual de análisis.

Para la simulación se ha definido el valor inicial de la estructura de costos así como ponderaciones de acuerdo al registro de transacciones para cada nivel de suministro. Se elaboró la tabla 1 para resumir la estructura de costos en MXN y valores iniciales.

Tabla 1

Costos totales del modelo de suministro

\begin{tabular}{lllll}
\hline & M & P & CD & Total \\
\hline Min (MXN) & 0,03 & 0,06 & 0,03 & 0,12 \\
Media (MXN) & 17,21 & 18,31 & 17,41 & 52,94 \\
Max (MXN) & 21,77 & 35,95 & 36 & 93,72 \\
Muestra & 1500 & 1500 & 1500 & 4500 \\
Ponderación (\%) & 23,22 & 38,35 & 38,41 & 100 \\
Valor (MXN) & 464575,33 & 767178,83 & 768 245,83 & 2000000 \\
\hline M: minorista, & P: proveedor, & CD: Centro de distribución & \\
\hline
\end{tabular}

Elaboración propia

De acuerdo al enfoque de Meucci (2010), se definió arbitrariamente un horizonte temporal entre los meses de junio y agosto (periodo con mayor volatilidad en la zona de estudio). Los registros que conforman la muestra representan 1500 días de actividad. La figura 2 ilustra el comportamiento de las transacciones para los tres niveles de suministro.

Para el estudio se ha transformado la serie de precios diarios en series de retorno logarítmico mediante la expresión 1. Muchos de los modelos de evaluación del riesgo se basan en la distribución normal de las series de datos. La rentabilidad logarítmica o capitalización compuesta continua se calcula usando logaritmos naturales; para este análisis se ha estimado también la volatilidad histórica para cada serie de retorno a partir de la ecuación 2.

$$
R_{t}=\ln \left[\frac{P_{t}}{P_{t-1}}\right]
$$




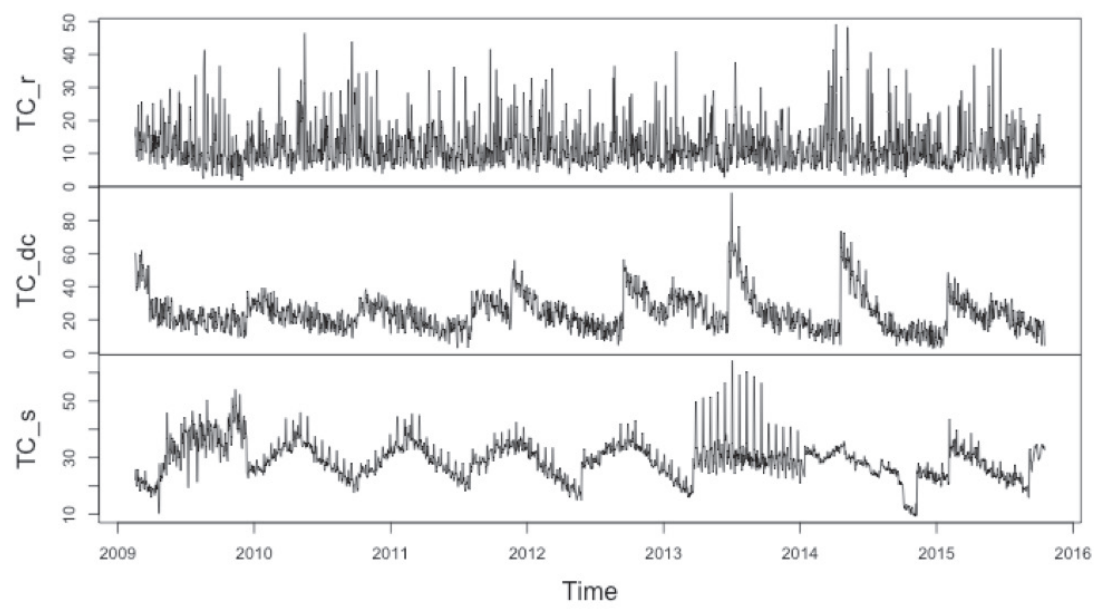

Figura 2. Volatilidad diaria por niveles de suministro

Elaboración propia

$$
\sigma=\sqrt{\frac{\sum_{i-1}^{T}\left(R_{i}-\mu\right)^{2}}{T-1}}, i=1,2,3, \ldots 1500
$$

Donde $\sigma$ es la desviación estándar de la muestra; T el número total de observaciones; $\mu$ la media del retorno de la serie y $\mathrm{R}_{\mathrm{i}}$ el retorno de los valores individuales.

La figura 3 describe el comportamiento temporal de los costos para cada nivel. Es posible apreciar en las figuras las fluctuaciones e incrementos súbitos en el horizonte temporal. Las dos entidades que resienten con mayor frecuencia las variaciones atípicas en la demanda son los minoristas (a) y los centros de distribución (b), donde se aprecian cambios importantes en el horizonte de análisis. En contraste, en los proveedores (c) se observa una tendencia creciente a lo largo del mismo periodo de análisis.

Se elaboró la tabla 2 para describir la matriz de correlaciones para los estados del sistema y para cada nivel de suministro. Los escenarios corresponden a los estadios entre las transacciones comerciales, siendo el estatus normal (N), entregas a tiempo (T), anticipadas (A) y con demora (D). La motivación ha sido estimar la pérdida potencial que podría sufrir el sistema considerando las condiciones referidas. En los últimos años el tema ha tenido un especial interés, por ejemplo en la evaluación del riesgo en cadenas de suministro (Heckmann et al., 2014; Freise y Seuring, 2015; Chopra y Sodhi, 2014; Piening, Ehrmann y Meiseberg, 2013). 


\section{Minoristas}

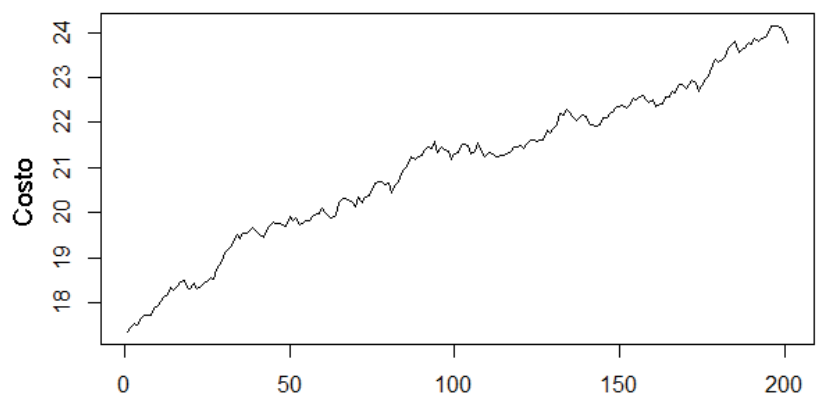

Centros de distribución

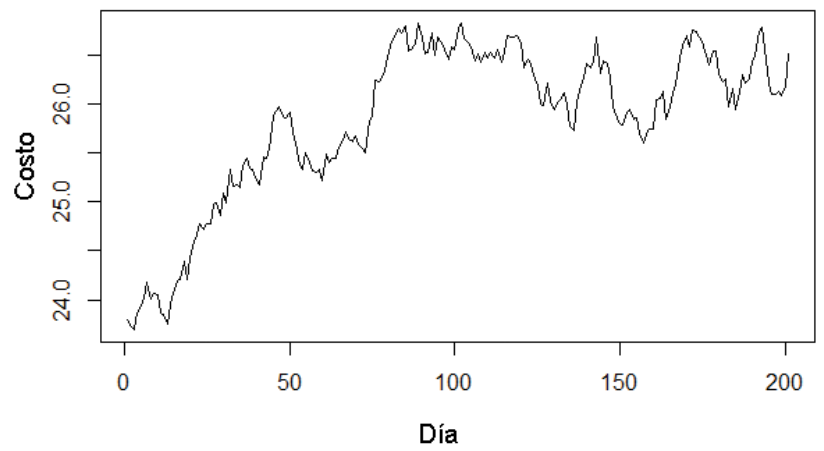

Proveedores

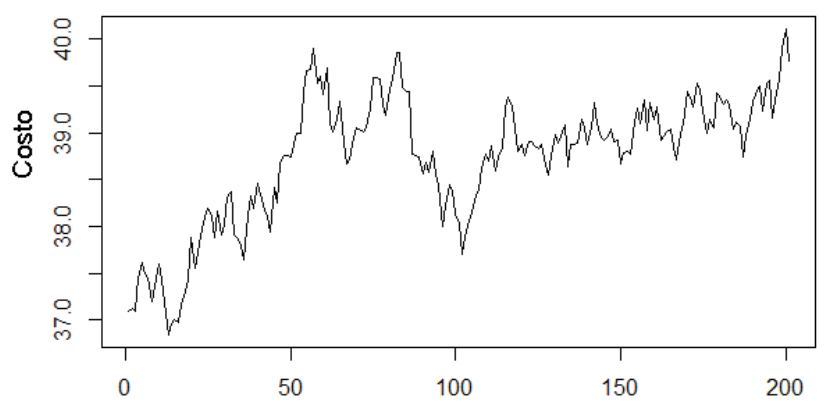

Figura 3. Análisis de la serie temporal de minoristas, centros de distribución y proveedores

Elaboración propia

Sin embargo, esta sección aborda la problemática desde una perspectiva en un entorno microsocial enmarcado por el aumento de la competencia, la minimización de costos de operación y el incremento de la eficiencia, entre otros factores. 
Otros estudios señalan la importancia de valorar los impactos ante contextos de riesgo y particularmente ante escenarios adversos asociados al clima, como los que han propuesto Jabareen (2013) y Rusman y Shimizu (2013).

Tabla 2

Resultados de salida de la matriz de correlaciones (\%)

\begin{tabular}{|c|c|c|c|}
\hline & $\begin{array}{l}\text { Centros de } \\
\text { distribución } \\
\text { (CD) }\end{array}$ & $\begin{array}{l}\text { Proveedores } \\
\text { (P) }\end{array}$ & $\begin{array}{c}\text { Minoristas } \\
\text { (M) }\end{array}$ \\
\hline$C D$ & 100 & 4,27 & 2,89 \\
\hline P & 4,27 & 100 & 6,93 \\
\hline M & 2,89 & 6,93 & 100 \\
\hline \multicolumn{4}{|c|}{ A } \\
\hline$C D$ & 100 & 2,61 & 1,23 \\
\hline$P$ & 2,61 & 100 & 2,30 \\
\hline M & 2,13 & 2,30 & 100 \\
\hline \multicolumn{4}{|c|}{$\mathrm{T}$} \\
\hline$C D$ & 100 & 14,02 & 0,57 \\
\hline$P$ & 14,02 & 100 & 0,54 \\
\hline M & 0,57 & 0,53 & 100 \\
\hline \multicolumn{4}{|c|}{ D } \\
\hline$C D$ & 100 & 2,59 & 1,15 \\
\hline P & 25,87 & 100 & 2,38 \\
\hline$M$ & 1,15 & 2,38 & 100 \\
\hline
\end{tabular}

Elaboración propia

Del total de la muestra se observa que el proveedor es un componente sensible en el sistema y que sus impactos trascienden hasta el nivel del centro de distribución en todos los escenarios. La relación mayor se observa en las transacciones con demora, es decir, en aquellos pedidos que no se realizaron en la fecha convenida $(25,85 \%)$, por lo que el nivel de riesgo es importante y significativo en el sistema.

\subsection{Valoración de escenarios mediante el enfoque Stress Test}

Esta sección propone un enfoque alterno para evaluar el comportamiento del valor en riesgo VaR mediante el método de Stress Test. A partir de los coeficientes de correlación para el horizonte mensual, se valoran los niveles máximos y así se discrimina la correlación observada en los tres estados. 
Un tratamiento importante, de acuerdo con Melnyk, Rodrigues y Ragatz (2009) y Alexander y Sheedy (2008), es la configuración de la matriz de correlación, que deberá ser positiva y cumplir con la propiedad matemática de Cholesky, por lo que se procedió al tratamiento de acuerdo a las siguientes expresiones:

$$
\begin{aligned}
& |\bar{\rho}|>0 \\
& A \cdot A^{T}=\bar{\rho}
\end{aligned}
$$

Donde $\rho$ es la matriz de correlación, $A$ la matriz de Cholesky y $A^{T}$ la matriz traspuesta de Cholesky.

A continuación describimos los planteamientos de las matrices de correlación, de acuerdo a las propiedades de la ecuación 3 , atendiendo la misma estructura de las variables descritas en la tabla de resultados de salida de la matriz de correlaciones (tabla 2). Para estimar el VaR se partió de la siguiente expresión:

$$
\operatorname{VaR}_{(\text {Stressed })}=\omega_{i} \cdot z \sigma_{i} \text {, daily }
$$

Donde $w_{i}$ es el valor inicial de las transacciones, $\sigma_{\mathrm{i}}$ daily expresa la volatilidad diaria de las transacciones asociadas al escenario disruptivo y $z$ el nivel de confiabilidad, igual a $-1,6449$.

Se ha estimado el VaR asociado a los escenarios de crisis, para lo cual se ha propuesto una magnitud alterna VaR para aproximarnos a los resultados reales al introducir los efectos de una diversificación en los datos de costo, en contraste con la estimación previa que nos arrojaron los resultados globales. Por lo tanto, para obtener los resultados alternos se partió de la siguiente expresión:

$$
\operatorname{VaR}_{c t}=\sqrt{V^{T} \cdot \vec{\rho} \cdot V}
$$

Un vector columna de dimensión $\mathrm{n} \times 1$ expresa los valores no diversificados del VaR; se ha estimado mediante el producto de $V=\sigma \bullet Z \bullet W$, que expresa el vector $V$ transpuesto y se estima a través del producto de:

$$
g V^{T}=W^{T} \cdot Z * \sigma
$$




\section{RESULTADOS}

\subsection{Enfoque de simulación de Monte Carlo}

Para esta sección hemos propuesto la simulación de Monte Carlo; un método no determinista o estadístico numérico usado para aproximar expresiones matemáticas complejas y difíciles de evaluar con exactitud (Rubinstein y Kroese, 2016). El planteamiento se obtiene mediante la siguiente expresión:

$$
C_{t}=C_{t-1} \cdot e^{\sigma \cdot z \sqrt{t}}
$$

Donde $C_{t}$ expresan los costos simulados, $C_{t-1}$ el costo actual, e, la variable aleatoria que se distribuye como una normal estandarizada con $\mu=0$ y 0 ? $=1$; ? la volatilidad diaria de la muestra, y $\sqrt{t}$, un factor ajustado que transforma la volatilidad diaria en horizontes temporales más amplios.

Por ejemplo, el VaR se calcula para un día; por lo tanto, su valor será igual a 1. En el caso de la selección de costos para los tres niveles de suministro, estarán representados por los costos unitarios por transacción.

Partiendo de este enfoque metodológico los números aleatorios normales deberán ser transformados en números aleatorios correlacionados de la matriz propuesta por Cholesky y mediante la expresión:

$$
\left[\begin{array}{l}
\varepsilon_{1} \\
\varepsilon_{2} \\
\varepsilon_{3}
\end{array}\right][A]=\left[\begin{array}{l}
Z_{r} \\
Z_{S} \\
Z_{d c}
\end{array}\right]
$$

Donde $\varepsilon$ definen el vector columna de las variables aleatorias que se distribuyen normalmente y $z$ es un vector de variables normales transformadas que contempla en su estructura la correlación histórica, siendo $A$ la matriz de estrés para cada escenario disruptivo.

Para la simulación se han definido 10000 costos correlacionados considerando la variable estrés-costos. Esto ha generado 10000 vectores $z$ con subíndices $i$. Este enfoque puede consultarse en los trabajos publicados por Fleming y Zizzo (2015), y Shekh y Marsh (2016). Las siguientes ecuaciones describen los parámetros de cálculo.

$$
\begin{aligned}
& C_{M}^{i}=23,80 \cdot e^{\sigma r \cdot z r}, i=1,2, \ldots, 10000 \\
& C_{P}^{i}=17,36 \cdot e^{\sigma s \cdot z s}, i=1,2, \ldots, 10000 \\
& C_{C D}^{i}=37,10 \cdot e^{\sigma d c-z d c}, i=1,2, \ldots, 10000
\end{aligned}
$$




\subsection{Resultados de salida}

Se ha elaborado la tabla 3 para resumir los resultados de las estimaciones del VaR diversificado.

Tabla 3

Resultados del análisis de escenarios

\begin{tabular}{lccc}
\hline & Minoristas & Proveedores & Centros de distribución \\
\hline Costo 1 (MXN) & 21,77 & 35,95 & 36,00 \\
Costo 2 (MXN) & 23,80 & 37,1 & 17,36 \\
Costo 3 (MXN) & 22,92 & 36,33 & 17,4 \\
Costo 4 (MXN) & 26,91 & 35,67 & 17,4 \\
Ponderación (\%) & 23,22 & 38,35 & 38,41 \\
Valor (MXN) & 464575,33 & 767178,83 & 768245,83 \\
Z (95 \%) & 1,64 & 1,64 & 1,64 \\
Volatilidad (\%) & 5,11 & 4,51 & 4,99 \\
\hline VaR 1 (MXN) & 850109,71 & 2046027,88 & 2270088,37 \\
VaR 2 (MXN) & 929380,39 & 2111478,01 & 1094687,06 \\
VaR 3 (MXN) & 895016,74 & 2067654,88 & 1097209,38 \\
VaR 4 (MXN) & 1050824,63 & 2030092,20 & 1097209,38 \\
\hline
\end{tabular}

Elaboración propia

Mediante las trayectorias aleatorias de los costos se ha estimado el valor global. Esto se obtiene mediante el producto entre el número de transacciones y los costos simulados.

También es posible evaluar para futuros análisis el cálculo de la ganancia esperada así como la distribución de pérdidas mediante la siguiente ecuación:

$$
L_{P}=W_{i}-W_{t}
$$

Donde $L_{P}$ es la distribución de pérdidas, el subíndice $P$ denota al proveedor, $W$ es el valor simulado del escenario $i$ y $W_{\mathrm{t}}$ el valor actual de acuerdo al horizonte temporal establecido.

\subsection{Análisis histórico y pruebas de estrés}

Finalmente se ha incorporado una variante alterna contemplando el aumento de la volatilidad. El interés ha sido valorar, mediante el enfoque de simulación, el comportamiento de los costos. Este responde a una selección de instantes asociados a sucesos 
disruptivos o, en su caso, a periodos con mayor volatilidad del comportamiento histórico de los costos (figura 3). El enfoque metodológico para esta valoración se fundamenta en los siguientes lineamientos: estimaciones

1. Selección del horizonte histórico de simulación, por lo cual se han propuesto 250 días.

2. Calcular el rendimiento de los costos históricos para cada nivel de suministro.

3. Finalmente, generar los costos históricos mediante la siguiente ecuación generalizada:

$C_{i}=C_{t-1} \cdot e^{R i}$,

Al sustituir las variables del proveedor (P) en la ecuación anterior, tendríamos que:

$P_{i}=P_{t-1} \cdot e^{R i}$,

Donde $P_{i}$ es el costo simulado para el escenario i del proveedor, siendo $i=1,2, \ldots, 19$; Pt-1 es el costo actual de la transacción del proveedor, y $R_{t-1}$ es la rentabilidad histórica del proveedor.

A partir de este tratamiento, el proceso es idéntico al descrito para la simulación de Monte Carlo y aplicable para los tres niveles de suministro. En la tabla 4 se han resumido todos los resultados para los tres niveles. La variable $p$ considera la estimación del nivel de pérdida más allá del estimador del VaR.

Tabla 4

Matriz de Cholesky para los tres niveles de suministro (MXN)

\begin{tabular}{rrrcc}
\hline Ítems & Minoristas & Proveedores & Centros de distribución & \multicolumn{1}{c}{ Total } \\
\hline Alpha $(0,05)$ & 25,81 & 18,84 & 40,28 & 84,93 \\
Alpha $(0,95)$ & 32,57 & 23,75 & 50,79 & 107,10 \\
Pérdida & 38720,63 & 28252,64 & 60416,49 & 382169,25 \\
\hline VaR & 48848,06 & 35620,40 & 76181,72 & 481950,50 \\
\hline
\end{tabular}

Elaboración propia

Mediante este enfoque se ha valorado el impacto para los tres niveles de suministro. La tabla 4 resume los costos asociados a las pérdidas potenciales para cada nivel. Como puede observarse, los efectos son mayores en los centros de distribución, en contraste con los detallistas y los proveedores. Evidentemente, están asociados al costo de las transacciones comerciales y al estado que guardan los productos a lo largo de la cadena. 
Las lecciones que se obtuvieron de los análisis están en la valoración del impacto de las pérdidas mediante simulación para un horizonte temporal determinado. Los resultados orientan a estudiar estrategias cuyos efectos puedan mejorar los niveles de servicio y, por ende, la satisfacción de los clientes en el sistema.

\section{CONCLUSIONES}

Esta investigación propuso el análisis del riesgo mediante el enfoque Stress Test bajo el paradigma de simulación de Monte Carlo. Los cálculos fueron programados e implementados mediante funciones en R-Studio.

La investigación surgió en relación a la diversificación de las soluciones mediante el enfoque de simulación. Se espera que los instrumentos tengan utilidad para la parametrización y calibración de futuros análisis en otros contextos de estudio. En general las aportaciones fueron: la generación de un marco conceptual y metodológico para la implementación de herramientas que valoren el riesgo del cumplimiento para un conjunto de transacciones comerciales, todas en el marco de un horizonte temporal caracterizado por efectos disruptivos, o en su caso escenarios con volatilidad en un horizonte temporal.

La base de datos refiere los registros de transacciones comerciales traducidas en costo por día entre los meses de junio y agosto, desde el 2009 al 2016. La muestra de transacciones comerciales para los tres niveles de suministro tiene un vínculo importante con los meses de mayor actividad asociados a eventos hidrometereológicos en la zona de estudio. Por ello ha sido de especial interés valorar el impacto del riesgo asociado a la volatilidad para un conjunto de escenarios.

Algunas consideraciones relevantes fueron: identificar los niveles de impacto en términos de costo para cuatro escenarios hipotéticos asociados a parámetros como los niveles de confiabilidad y la volatilidad en un horizonte temporal de riesgo típico en zonas con afectaciones cuyos efectos derivan en unas fluctuaciones en la demanda conocidos como "efecto látigo".

Es importante concluir que los efectos y costos asociados son amortizados con características diferentes y en instantes temporales desfasados, haciendo referencia al efecto látigo estudiado por Hussain (2012) y Buchmeister, Pavlinjek, Palcic y Polajnar (2008).

La propuesta reconfigura el modelo conceptual tradicional, cuya relación intrínseca, situado en un contexto sometido a procesos hidrometereológicos de riesgo, dota de complejidad al sistema. Además de la exploración del riesgo propusimos la modelación y, mediante el paradigma de simulación de Monte Carlo, evaluamos los efectos de un cambio en el estado del suministro y patrones de respuesta. 
Para la evaluación experimental se implementaron enfoques para la gestión del riesgo en un caso de estudio. Para ello se configuraron cuatro escenarios de respuesta que responden y están estrechamente vinculados a la fase de exploración del riesgo. También incorporamos una solución basada en la prueba de estrés con parámetros asociados con los niveles de confiabilidad y volatilidad para horizontes temporales con características y patrones afines a los meses de mayor actividad climática en la zona de estudio.

El enfoque que se llevó a cabo valora los efectos disruptivos y los costos asociados con base en el VaR paramétrico, que ha demostrado ser más sensible mediante las pruebas de estrés. Con la condición de introducir un tratamiento previo para verificar la coherencia interna de los datos, es decir, verificar que la matriz de correlaciones sea positiva, es posible resolver este tratamiento descomponiendo sus factores por el método de Cholesky. Fue posible concluir que este procedimiento ofrece soluciones mucho más eficientes mediante el enfoque de simulación de Monte Carlo.

\section{REFERENCIAS}

Abdymomunov, A. y Gerlach, J. (2014). Stress testing interest rate risk exposure. Journal of Banking \& Finance 49, pp. 287-301. doi: 10.1016/j.jbankfin.2014.08.013

Alexander, C. y Sheedy, E. (2008). Developing a stress testing framework based on market risk models. Journal of Banking \& Finance 32(10), pp. 2220-2236. doi:10.1016/j. jbankfin.2007.12.041

Altiok, T. y Melamed, B. (2007). Simulation Modeling and Analysis with ARENA. Amsterdam: Elsevier.

Boucher, C. M., Daníelsson, J., Kouontchou, P. S. y Maillet, B. B. (2014). Risk models-atrisk. Journal of Banking \& Finance 44, pp. 72-92.

Buchmeister, B., Pavlinjek, J., Palcic, I. y Polajnar, A. (2008). Bullwhip effect problem in supply chains. Advances in Production Engineering and Management 3(1), pp. 45-55.

Bueno-Solano, A. y Cedillo-Campos, M. G. (2014). Dynamic impact on global supply chains performance of disruptions propagation produced by terrorist acts. Transportation Research Part E: Logistics and Transportation Review 61, pp. 1-12. doi: 10.1016/j.tre.2013.09.005

Chopra, S. y Sodhi, M. S. (2014). Reducing the Risk of Supply Chain Disruptions. MIT Sloan Management Review 55(3), pp. 73-80.

Escalante, J., Monsreal, I. y Casanovas, J. (Eds.). (2016). Logistics Practices in Small and Medium Enterprises (SME): Risk Context Survey for Hurricanes. En: Hernández, 
G. A., Sánchez-Ramírez, C. y García-Alcaraz, J. L. (Eds.), Handbook of Research on Managerial Strategies for Achieving Optimal Performance in Industrial Processes. Hershey: IGI Global, pp. 76-99.

Flood, M. D. y Korenko, G. G. (2015). Systematic scenario selection: stress testing and the nature of uncertainty. Quantitative Finance 15(1), pp. 43-59.

González Diez, V. M., Verner, D., Corrales, M. E., Puerta, J. M., Mendieta Umaña, M. P., Morales, C., ... L'Hoste, M. (2014). Climate change at the IDB: Building resilience and reducing emissions. Washington: Banco Interamericano de Desarrollo.

Heckmann, I., Comes, T. y Nickel, S. (2014). A Critical Review on Supply Chain Risk -Definition, Measure and Modeling. Omega 52, pp. 119-132. doi: 10.1016/j. omega.2014.10.004

Hernández, G. A., Sánchez-Ramírez, C. y García-Alcaraz, J. L. (2016). Handbook of Research on Managerial Strategies for Achieving Optimal Performance in Industrial Processes. Hershey: IGI Global.

Hussain, M. (2012). Quantifying the impact of a supply chain's design parameters on the bullwhip effect using simulation and Taguchi design of experiments. International Journal of Physical Distribution \& Logistics Management 42(10), pp. 947-968.

Instituto Nacional de Estadística y Geografía de México (INEGI) (2009). Micro, pequeña, mediana y gran empresa. Estratificación de los establecimientos. Censos económicos. Recuperado de https://www.inegi.org.mx/default.html

Instituto Nacional de Estadística y Geografía de México (INEGI) (2014). Censos económicos 2014. Recuperado de https://www.inegi.org.mx/default.html

Jabareen, Y. (2013). Planning the resilient city: Concepts and strategies for coping with climate change and environmental risk. Cities 31, pp. 220-229. doi: 10.1016/j. cities.2012.05.004

Jorion, P. (2010). Risk Management. Annual Review of Financial Economics 2, pp. 347-365

Kelton, W. D., Sadowski, R. P. y Sturrock, D. T. (2008). Simulación con software Arena. México: McGraw-Hill.

Melnyk, S. A., Rodrigues, A. y Ragatz, G. L. (2009). Using simulation to investigate supply chain disruptions. En: Zsidisin, G. A. y Ritchie, B. (Eds.). Supply Chain Risk. Boston: Springer, pp. 103-122.

Meucci, A. (2010). Historical scenarios with fully flexible probabilities. GARP Risk Professional, pp. 47-51.

Olson, D. L. y Wu, D. D. (2017). Enterprise Risk Management Models. Berlín: Springer. 
Rubinstein, R. Y. y Kroese, D. P. (2016). Simulation and the Monte Carlo method. New Jersey: John Wiley \& Sons.

Rusman, M. y Shimizu, Y. (2013). Effect of Continuity Rate on Multistage Logistic Network Optimization under Disruption Risk. Industrial Engineering and Management Systems 12(2), pp. 74-84. doi: 10.7232/iems.2013.12.2.074

Shekh, S. y Marsh, L. (2016). The Twenty-Ninth International Flairs Conference. Edimburgo. 
